Гринфельд Г. М., Голоколос Д. А.

ПАРАМЕТРИЧЕСКАЯ КОРРЕКЦИЯ СИСТЕМЫ УПРАВЛЕНИЯ ГЕРМЕТИЧНОГО ЭЛЕКТРОМЕХАНИЧЕСКОГО

ПРЕОБРАЗОВАТЕЛЯ

Гринфельд Г. М., Голоколос Д. А.

G. M. Grinfeld, D. A. Golokolos

ПАРАМЕТРИЧЕСКАЯ КОРРЕКЦИЯ СИСТЕМЫ УПРАВЛЕНИЯ ГЕРМЕТИЧНОГО ЭЛЕКТРОМЕХАНИЧЕСКОГО ПРЕОБРАЗОВАТЕЛЯ

\title{
PARAMETRIC CORRECTION OF HERMETIC ELECTROMECHANICAL CONVERTER CONTROL SYSTEM
}

Гринфельд Григорий Михайлович - кандидат технических наук, доцент кафедры «Электропривод и автоматизация промышленных установок» Комсомольского-на-Амуре государственного технического университета (Россия, Комсомольск-на-Амуре); 681018, г. Комсомольск-на-Амуре, ул. Пионерская, 61-20; тел. +7 (914) 1743914. E-mail: grinfelds2002@mail.ru.

Mr. Grigory M. Grinfeld - PhD, Associate Professor, Department of Electric Drive and Automation of Industrial Plants, Komsomolsk-on-Amur State Technical University (Russia, Komsomolsk-on-Amur); 681018, Komsomolskon-Amur, Pionerskaya str., 61-20; tel.+7(914)1743914. E-mail: grinfelds2002@mail.ru.

Голоколос Дмитрий Анатольевич - аспирант кафедры «Электромеханика» Комсомольского-на-Амуре государственного технического университета (Россия, Комсомольск-на-Амуре); Россия, 681024, г. Комсомольск-на-Амуре, пр. Интернациональный, д. 5, кв. 31; тел. 8 (914) 2123472. E-mail: dag@land.ru.

Mr. Dmitry A. Golokolos - PhD candidate, Department of Electromechanics, Komsomolsk-on-Amur State Technical University (Russia, Komsomolsk-on-Amur); house 59, flat 31, International av., Komsomolsk-on-Amur, Russia; tel. 8 (914) 2123472. E-mail: dag@land.ru.

Аннотация. В статье приведена методика синтеза регуляторов системы управления, учитывающая специфические особенности конструкции герметичных электромеханических преобразователей. Целью исследования является выработка принципов параметрической коррекции, позволяющих встраивать герметичные электромеханические преобразователи в действующие системы управления.

Summary. The article presents the method of control system regulator synthesis, considering peculiarities of hermetic electromechanical converters. The purpose of the study is to estimate the parametric correction conceptions, admitting to embed hermetic electromechanical converters in existing control system.

Ключевые слова: система управления, герметичный электромеханический преобразователь, капсулированный электродвигатель.

Key words: control system, hermetic electromechanical converter, canned motor.

УДК 621.313

\section{Введение}

Герметичные электромеханические преобразователи (ГЭМП) предназначены для применения в качестве приводов при работе в сложных условиях эксплуатации: на огнеопасных объектах, при высоких и низких давлениях и температурах, в обстановке повышенной влажности и вибрации. Характерным отличительным элементом таких электродвигателей является наличие экранирующей гильзы, устанавливаемой в зазор между статором и ротором и защищающей обмотки и магнитопровод статора от воздействия рабочей среды.

Указанная конструктивная особенность ГЭМП, наряду с рядом других, обуславливает значительную специфику протекающих в них электромагнитных и тепловых процессов.

В [1] отмечено значительное влияние экранирующего элемента ГЭМП на его рабочие характеристики. При этом в качестве определяющего фактора рассматривается изменение величины индуктивного сопротивления экрана при работе ГЭМП в составе электротехнического комплекса, т.к. при питании от преобразователя частоты его влияние может быть значительным. 
Для эффективного решения и нахождения параметров необходима многомерная параметризация объекта исследования с использованием возможностей CAПР-технологий (SOLIDWORKS, T-FLEX CAD) и численное моделирование на основе мультифизичных программ (ANSYS, MAXWELL, FEMLAB, FLUENT, ELCUT, COMSOL). Указанные программные комплексы позволяют найти эмпирические выражения для расчета параметров экрана при проектировании электромеханических преобразователей.

Системы управления современных преобразователей частоты с эталонной математической моделью объекта управления могут аналитически рассчитать и регулировать выходные параметры на основе значений фазных токов и потокосцеплений электромеханического преобразователя, но поддержание устойчивой скорости вращения в области малых частот, обеспечение номинального момента при нулевой скорости, робастность, поддержание энергосберегающего режима работы возможны лишь при определенных параметрах модели [2]. Кроме того, существующие преобразователи частоты не позволяют задать параметры экранирующего элемента напрямую даже при возможности их расчета, что ведёт к расхождению характеристик эталонной математической модели в преобразователе частоты и математической модели реального ГЭМП. К числу дополнительных усложняющих факторов следует отнести существенную нелинейность электромеханического преобразователя как объекта управления, дискретность процессов, протекающих в преобразователе частоты, необходимость компенсации внутренних перекрестных связей объекта управления.

\section{Математическая модель объекта управления}

Пространственная модель герметичного асинхронного электродвигателя на основе уравнений обобщенного электромеханического преобразователя [3] может быть представлена в следующем виде (см. рис. 1).

Обозначения, принятые на рис. 1:

$L_{A}, L_{B}, L_{C}-$ полные индуктивности фаз обмотки статора;

$L_{A_{e c}}, L_{B e c}, L_{C_{e c}}$ - полные приведенные индуктивности фиктивной обмотки вихревых токов, учитывающей потери в стали;

$L_{A_{x}}, L_{B_{x}}, L_{C_{x}}-$ полные приведенные индуктивности экрана фаз;

$L_{a}, L_{b}, L_{c}$ - полные приведенные индуктивности соответствующих фаз обмотки ротора;

$\gamma$ - угол поворота ротора, электрический радиан.

При разработке математической модели были приняты следующие допущения:

1. трехфазная система симметрична, нулевой ток в ней отсутствует, сумма мгновенных значений токов фаз равна нулю;

2. каждый протекающий по фазной обмотке ток порождает магнитодвижущую силу, синусоидально распределенную по окружности воздушного зазора машины;

3. учитывается только основная гармоническая составляющая всех известных и определяемых величин;

4. влияние гистерезиса стали на форму токов и потокосцеплений двигателя пренебрежительно мало;

5. индукция магнитного поля в лобовых частях обмоток равна нулю;

6. магнитное поле, связанное с экранирующим элементом и вторичной вращающейся короткозамкнутой обмоткой, ограничено расчетной длиной воздушного зазора, равномерно по длине зазора и имеет только нормальную составляющую, неизменную по всей толщине обмоток и гармонически изменяющуюся по окружности воздушного зазора;

7. магнитная проницаемость экрана равна магнитной проницаемости зазора.

Представленную физическую модель в естественных координатах описывает следующая система уравнений, записанная в матричном виде: 


$$
\left\{\begin{array}{c}
{[U]=[R] \cdot[I]+\frac{d}{d t}[\Psi],} \\
{[\Psi]=[L] \cdot[I],} \\
M_{\ni \mathrm{M}}=\frac{1}{2}\left([I]^{T} \cdot \frac{d}{d y}[L] \cdot[I]\right), \\
\frac{d \omega_{p}}{d t}=\frac{1}{J}\left(M_{\text {эм }}-M_{\mathrm{c}}\right),
\end{array}\right.
$$

где $[U]$ - вектор напряжений; $[R]$ - матрица активных сопротивлений обмоток; $[I]$ - вектор токов;

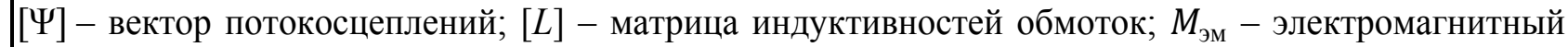
момент; $\omega$ - частота вращения ротора; $M_{\mathrm{c}}$ - момент сопротивления на валу.

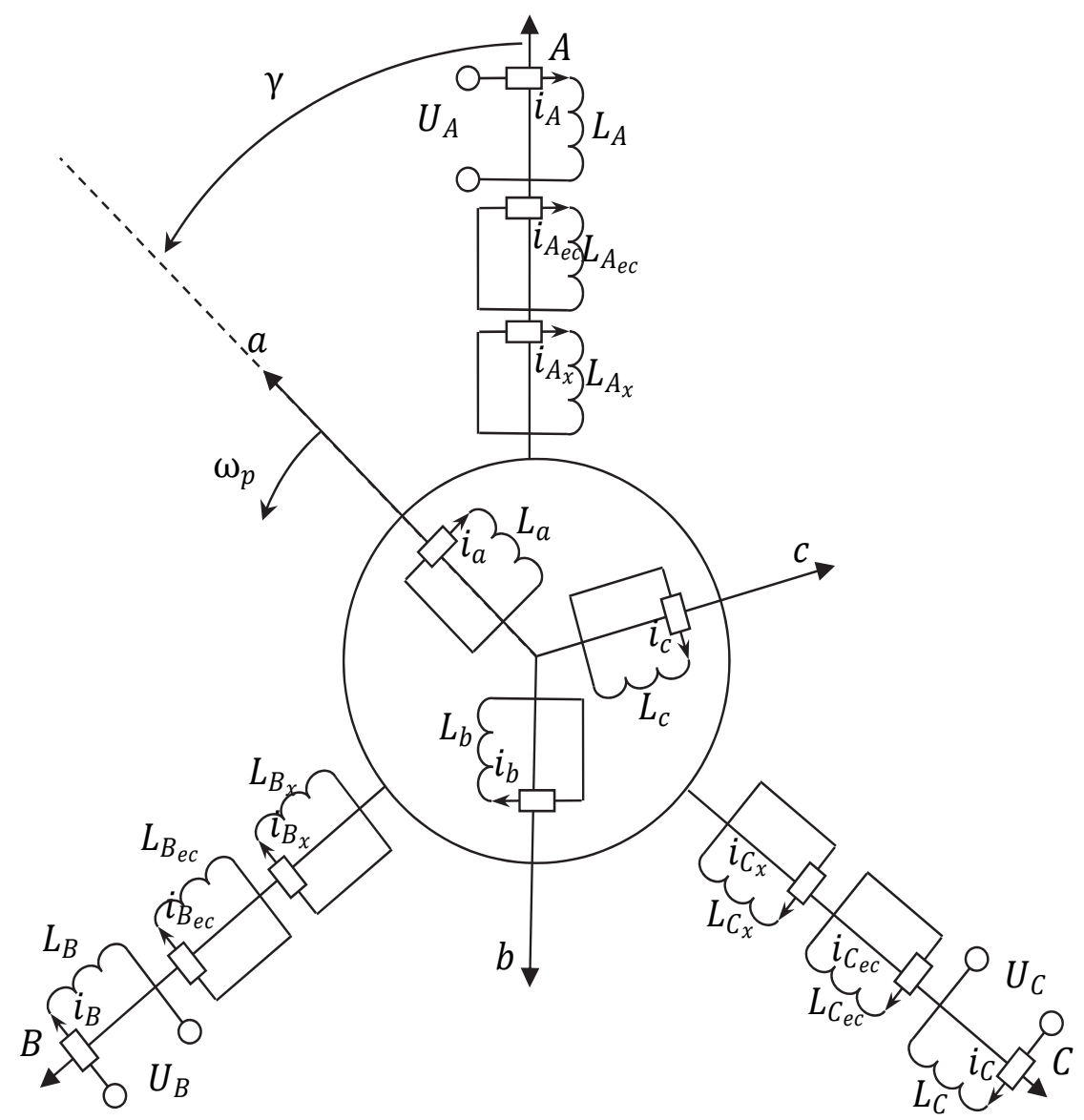

Рис. 1. Пространственная модель приведенного капсулированного электродвигателя в естественной вращающейся системе координат

В матрицу индуктивностей $[L]$ входит 16 субматриц. За исключением четырех диагональных субматриц полных индуктивностей, остальные отражают связи между статорными и роторными обмотками. При условии, что фазы электрической машины симметричны, матрица индуктивностей выглядит следующим образом:

$$
[L]=\left[\begin{array}{cccc}
{\left[L_{s}\right]} & {\left[L_{s r}\right]} & {\left[L_{\text {s.ec }}\right]} & {\left[L_{s x}\right]} \\
{\left[L_{r s}\right]} & {\left[L_{r}\right]} & {\left[L_{r . e c}\right]} & {\left[L_{r x}\right]} \\
{\left[L_{e c . s}\right]} & {\left[L_{e c . r}\right]} & {\left[L_{e c}\right]} & {\left[L_{e c . x}\right]} \\
{\left[L_{x s}\right]} & {\left[L_{x r}\right]} & {\left[L_{x . e c}\right]} & {\left[L_{x}\right]}
\end{array}\right],
$$




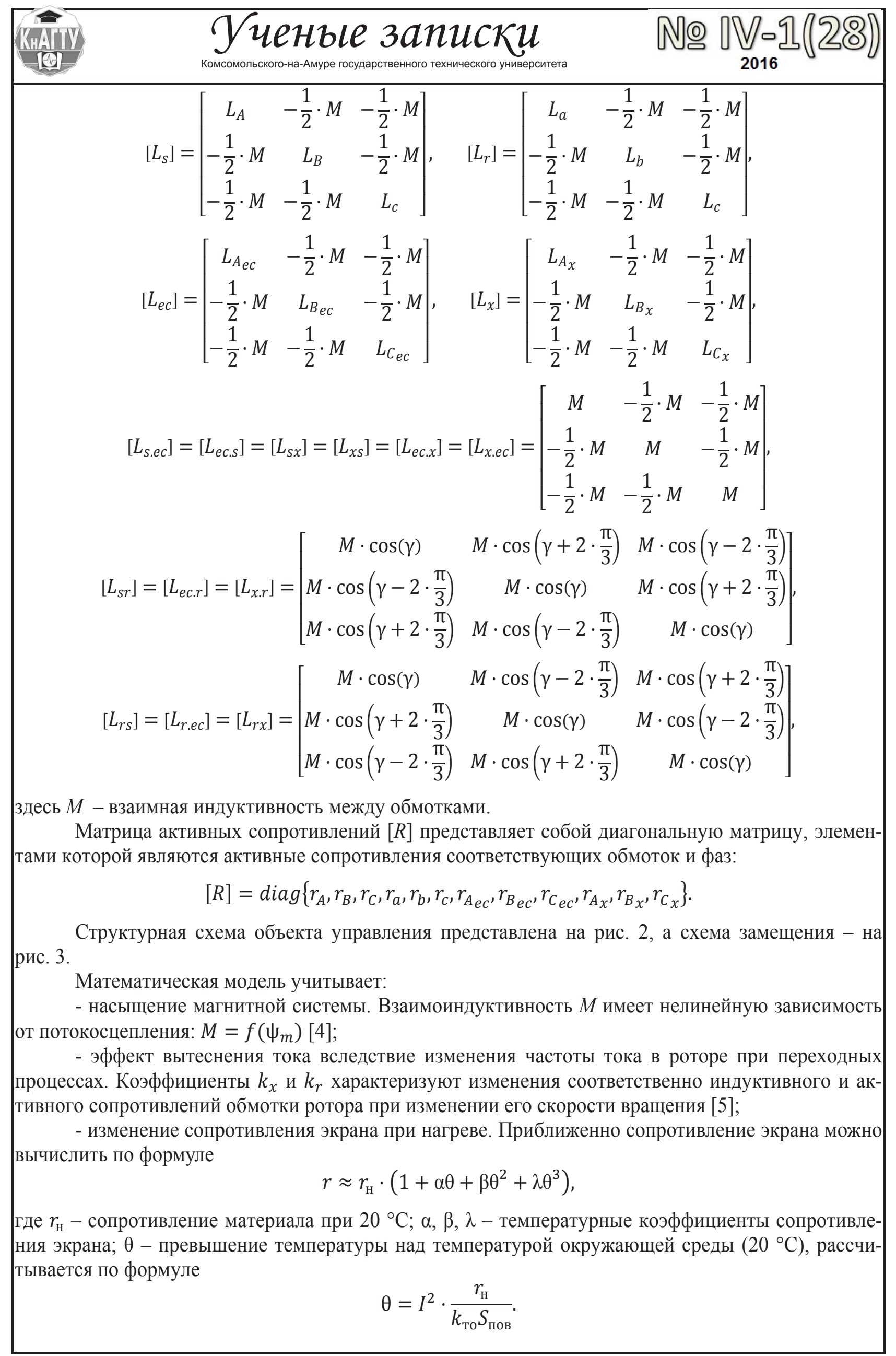


Гринфельд Г. М., Голоколос Д. А.

ПАРАМЕТРИЧЕСКАЯ КОРРЕКЦИЯ СИСТЕМЫ УПРАВЛЕНИЯ ГЕРМЕТИЧНОГО ЭЛЕКТРОМЕХАНИЧЕСКОГО

ПРЕОБРАЗОВАТЕЛЯ

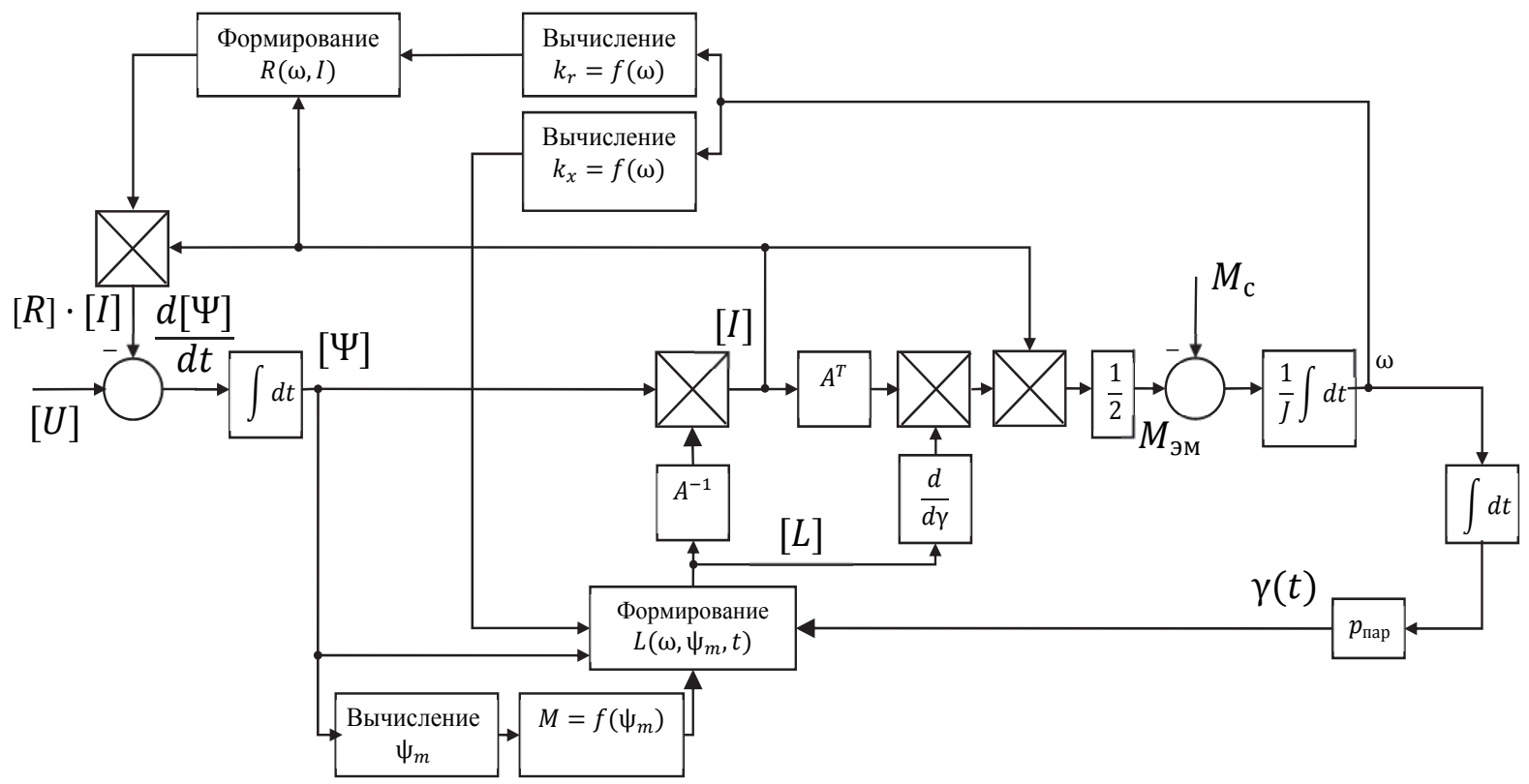

Рис. 2. Структурная схема герметичного асинхронного двигателя (АД): $p_{\text {пар }}$ - число пар полюсов электрической машины

Представленная модель позволяет исследовать ГЭМП как в установившихся, так и переходных режимах работы. К числу исходных параметров, необходимых для ее формирования, относятся первичные напряжения и конструктивные параметры устройства (геометрические размеры, обмоточные данные и т.п.). Модель учитывает существенную нелинейность и нестационарность объекта управления: коэффициенты модели, связанные с определяемыми величинами (активные сопротивления, индуктивности, взаимные индуктивности, определяемые расчетным или опытным путем), являются нелинейными функциями этих величин и зависят от выходных параметров объектов управления (ОУ).

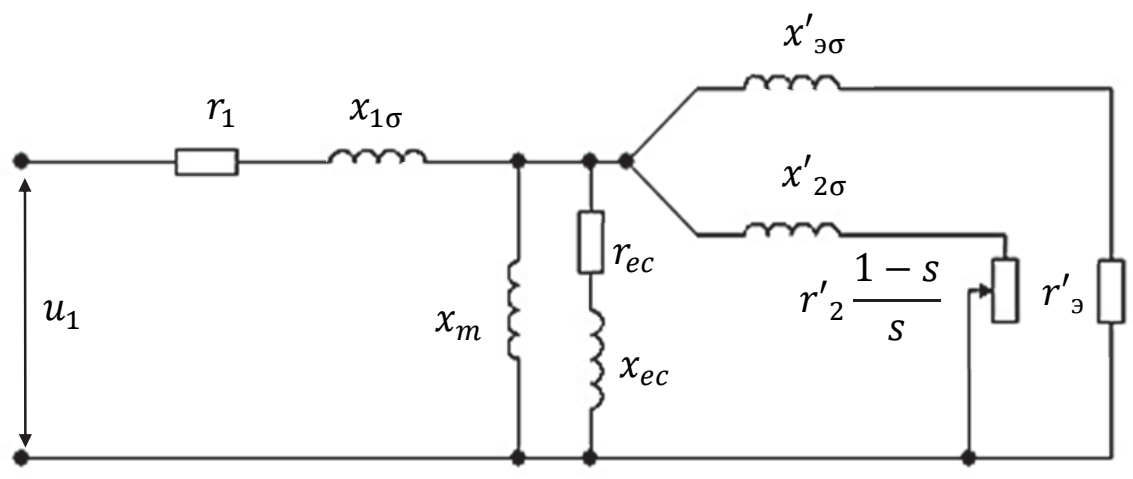

Рис. 3. Схема замещения герметичного АД

На рис. 4 приведены характеристики пуска на холостом ходу и наброса нагрузки ГЭМП, полученные при решении полевой задачи методом конечных элементов в Ansys Maxwell и при моделировании в Simulink MATLAB (1).

Сравнение приведенных графиков отражает достаточно высокую степень адекватности математической модели ГЭМП, погрешность пусковых характеристик составляет не более $2 \%$.

\section{Система управления герметичного электромеханического преобразователя}

Система автоматического управления электромеханического преобразователя обычно обеспечивает скорости и/или положения рабочего органа, тока или момента электропривода. До- 
биться желаемых выходных характеристик позволяет система с прямым управлением моментом (DTC). Подобные системы управления хорошо изучены и освещены в литературе [4; 6; 7]. Типичная схема системы DTC приведена на рис. 5.
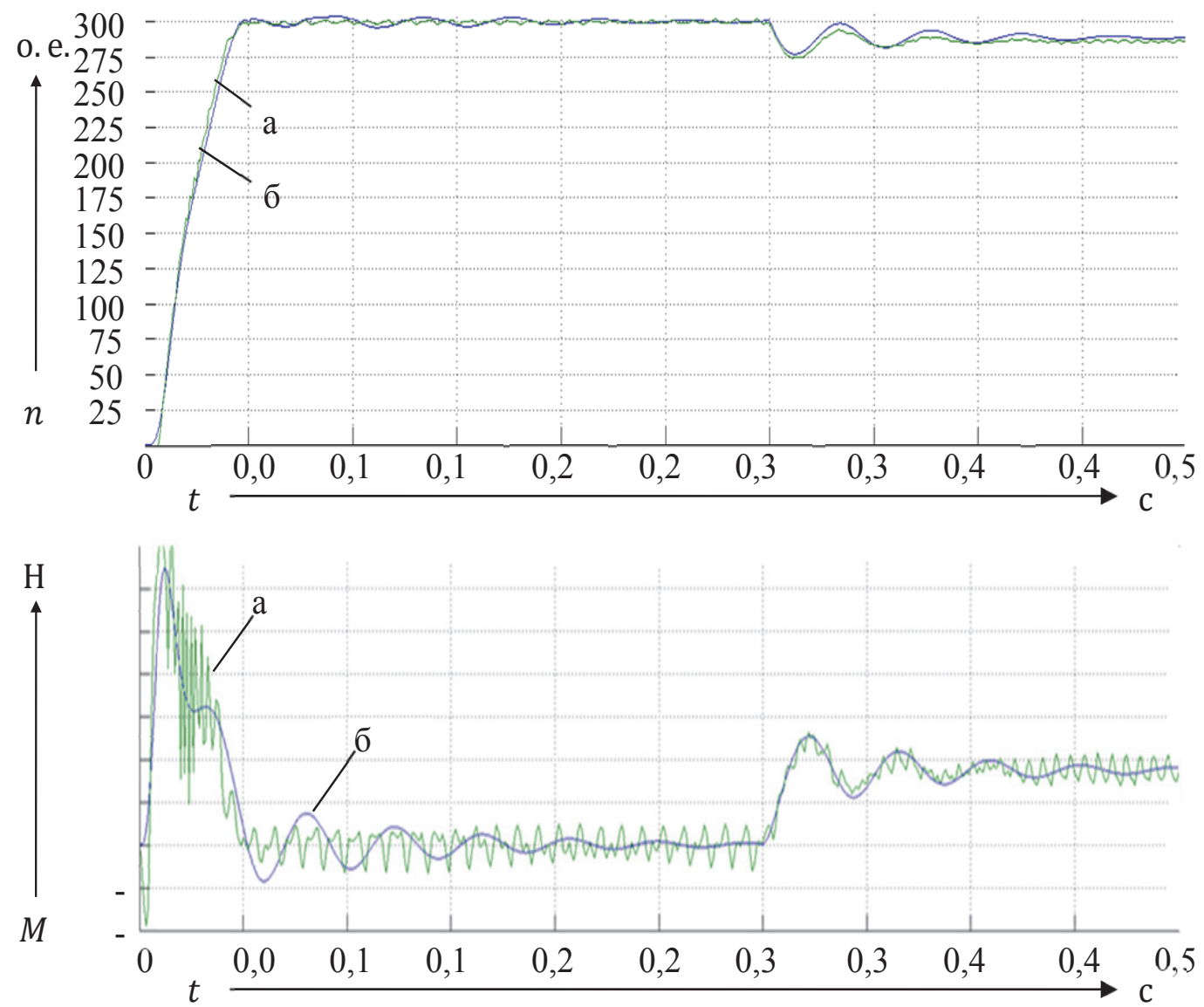

Рис. 4. Динамические характеристики скорости $n$ и момента $M$ пуска и наброса нагрузки герметичного АД мощностью 1 кВт:

a - полученные при решении полевой задачи в Ansys Maxwell; б - полученные при математическом моделировании в Simulink MATLAB

Работа систем DTC предполагает наличие информации о пространственном положении вектора потокосцепления, получаемой на практике от наблюдателя магнитного потока, обеспечивающего асимптотическую оценку этой переменной. Достоверность работы такого наблюдателя зависит от точности определения параметров ОУ. Как видно из рис. 6, механическая характеристика герметичного АД отличается от АД без экранирующего элемента даже при малых мощностях двигателя. Эта разница усиливается при повышении мощности двигателей, т.к. активное сопротивление экрана уменьшается, что приводит к росту вихревых токов и демпфирующего эффекта экранирующего элемента, который приводит к увеличению критического скольжения [1].

Таким образом, система управления, реализованная с использованием существующих преобразователей частоты, не может эффективно управлять ГЭМП, т.к. в её логике заложена другая математическая модель ОУ. бами:

Повышение эффективности работы системы управления с ГЭМП возможно двумя спосо-

1. путём преобразования структуры математической модели ГЭМП, заложенной в программу преобразователя частоты, к виду, учитывающему наличие экранирующего элемента;

2. с помощью параметрической коррекции существующей эталонной модели, реализуемой на основе приведения схемы замещения герметичного АД к эквивалентной, как показано на рис. 7. 
Гринфельд Г. М., Голоколос Д. А.

ПАРАМЕТРИЧЕСКАЯ КОРРЕКЦИЯ СИСТЕМЫ УПРАВЛЕНИЯ ГЕРМЕТИЧНОГО ЭЛЕКТРОМЕХАНИЧЕСКОГО ПРЕОБРАЗОВАТЕЛЯ

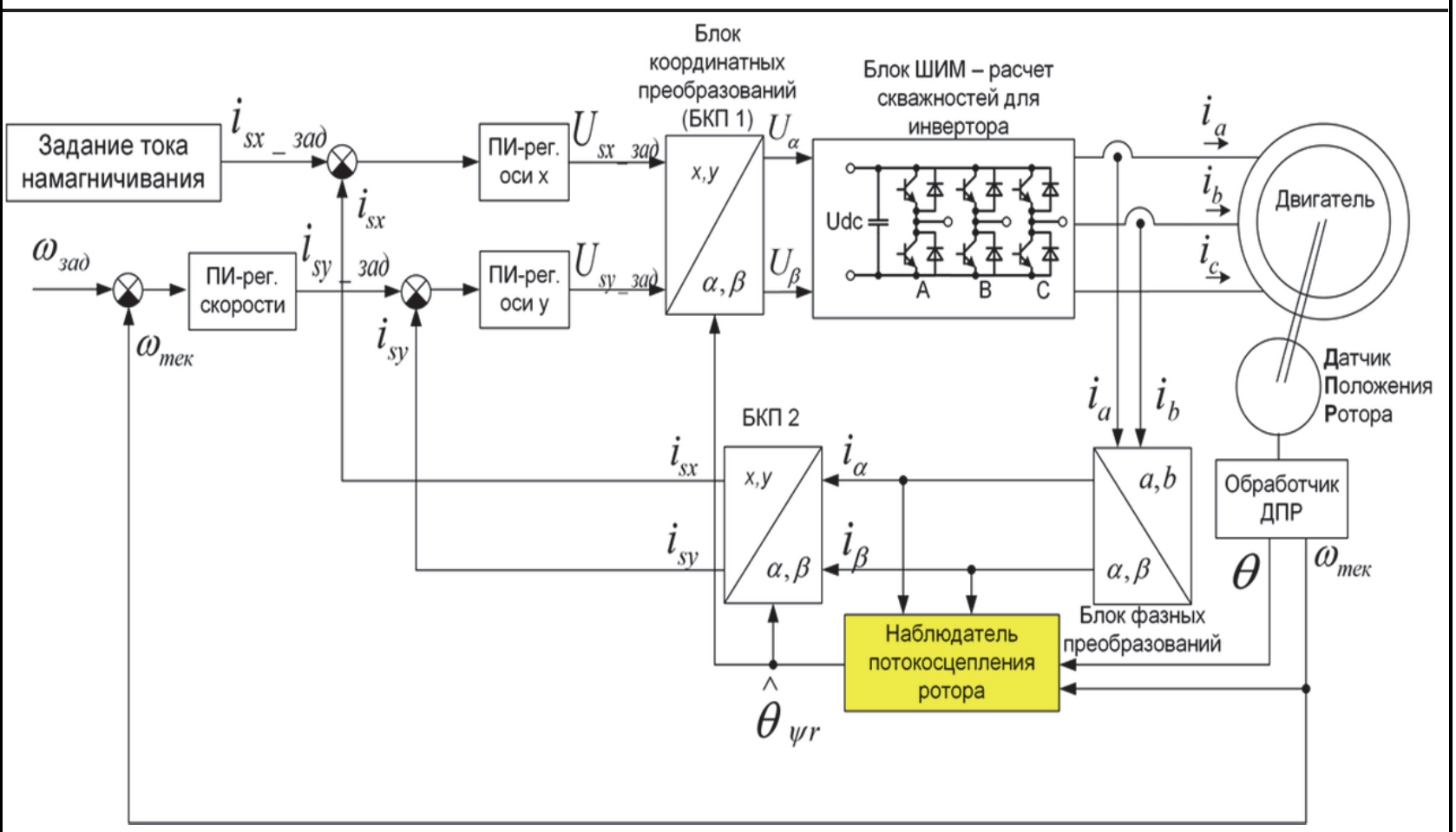

Рис. 5. Структурная схема системы с прямым управлением моментом (DTC)

Очевидно, что предпочтительным является второй способ, т.к. он даёт возможность использовать герметичные АД в существующих и уже работающих системах управления.

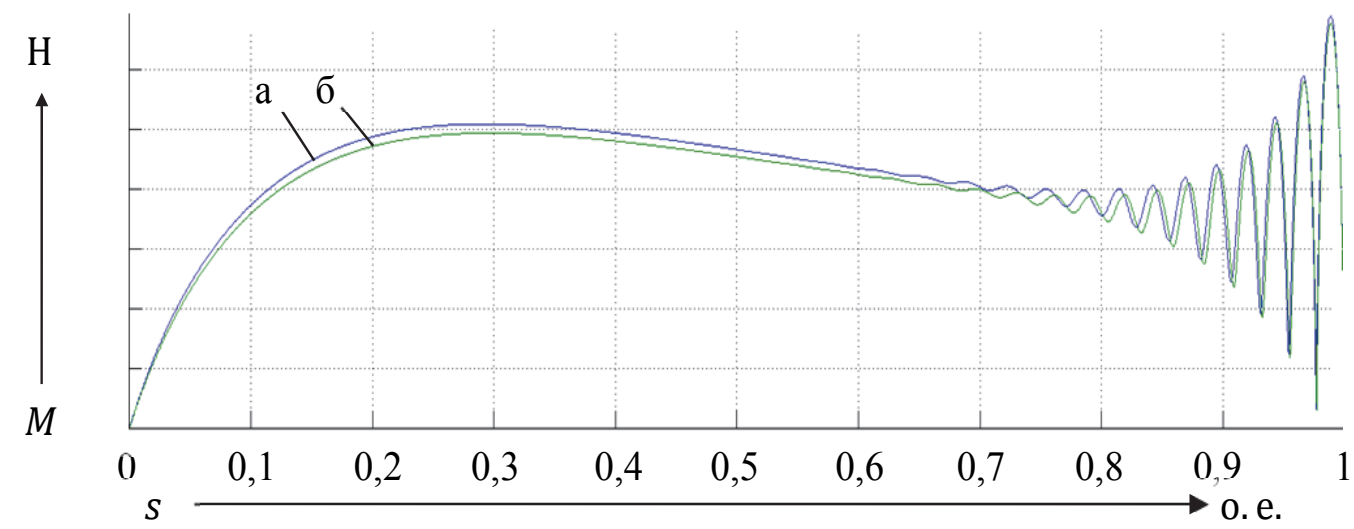

Рис. 6. Механическая характеристика двигателя мощностью 1 кВт: а - асинхронного двигателя без экранирующего элемента; б - герметичного асинхронного двигателя

Обозначим пусковые характеристики скорости и момента АД без экранирующего элемента $\omega=f(t)$ и $M=f(t)$, а характеристики герметичного АД соответственно $\omega_{\ni}=f(t)$ и $M_{\ni}=f(t)$. Переменные, приведенные в относительных единицах, отметим индексом *.

Для параметрической коррекции математической модели системы управления необходимо определить оптимальные параметры эквивалентной схемы замещения $r_{1 э}, x_{1 э \sigma}, x_{m э}$. Решение этой задачи сводится к нахождению экстремума (минимума) квадратичного интегрального критерия:

$$
f\left(r_{1 \ni}, x_{1 \ni \sigma}, x_{m \ni}\right)=\int \omega_{\Delta}(t)^{2} d t+\int M_{\Delta}(t)^{2} d t,
$$

где $\omega_{\Delta}(t)=\omega_{* \ni}(t)-\omega_{*}(t) ; M_{\Delta}(t)=M_{* \ni}(t)-M_{*}(t)$.

Выражение (2) представляет собой сумму квадрата ошибок пусковых характеристик герметичного АД и АД без экранирующего элемента. 


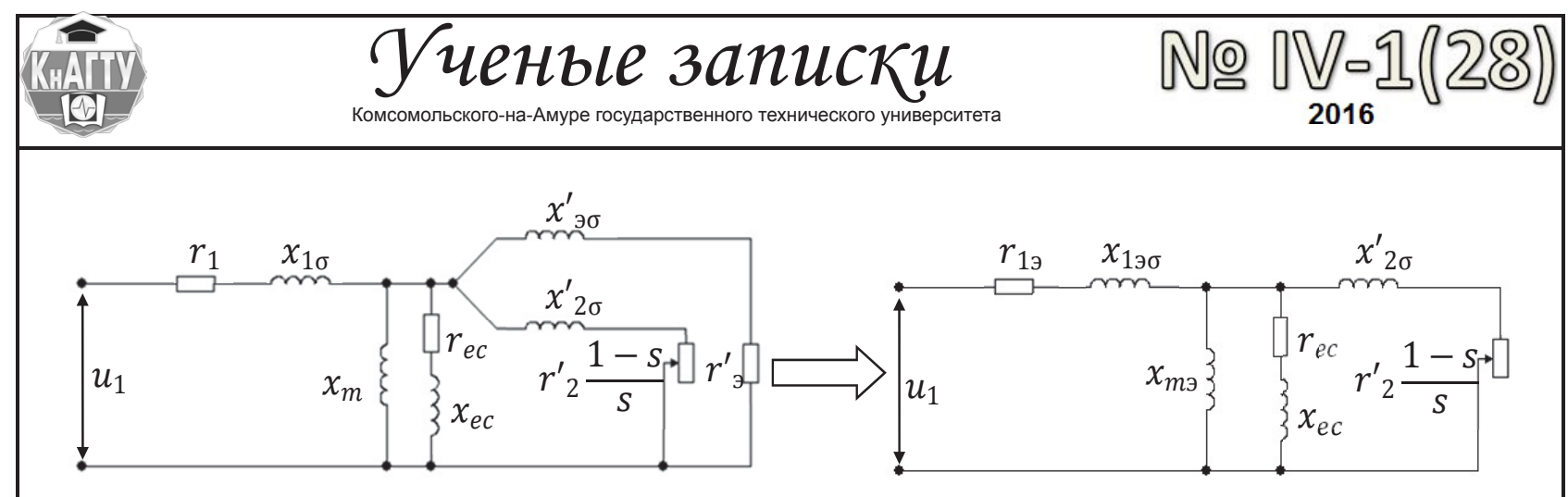

Рис. 7. Приведение схемы замещения герметичного АД к эквивалентной

Задача оптимизации может быть решена численно с помощью метода градиентного спуска:

$$
\begin{aligned}
& r_{1 \ni}^{[i+1]}=r_{1 \ni}^{[i]}-\lambda^{[i]} \nabla f\left(r_{1 \ni}^{[i]}, x_{1 \ni \sigma}^{[i]}, x_{m \ni}^{[i]}\right), \\
& x_{1 \ni \sigma}^{[i+1]}=x_{1 \ni \sigma}^{[i]}-\lambda^{[i]} \nabla f\left(r_{1 \ni}^{[i]}, x_{1 \ni \sigma}^{[i]}, x_{m \ni}^{[i]}\right), \\
& x_{m \ni}^{[i+1]}=x_{m \ni}^{[i]}-\lambda^{[i]} \nabla f\left(r_{1 \ni}^{[i]}, x_{1 \ni \sigma}^{[i]}, x_{m \ni}^{[i]}\right) .
\end{aligned}
$$

где $i$ - номер итерации; $\lambda^{[i]}$ - шаг на $i$-й итерации.

Полученные оптимальные значения $r_{1 э}, x_{1 э \sigma}, x_{m э}$ задаются в преобразователе частоты системы управления. Динамические пусковые характеристики АД в составе системы DTC приведены на рис. 8.

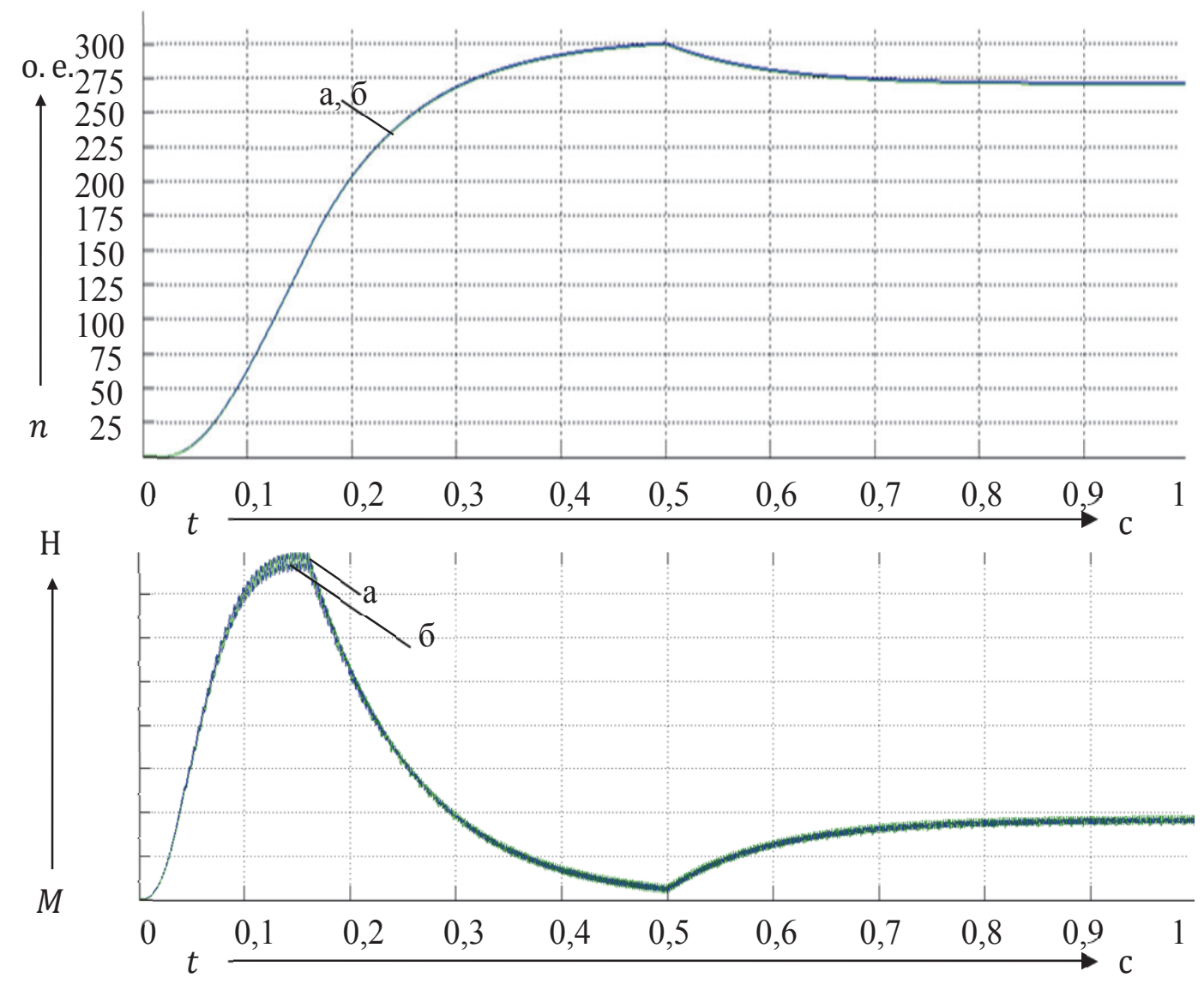

Рис. 8. Пуск и наброс нагрузки при номинальной частоте вращения в системе DTC: a - асинхронного двигателя без экранирующего элемента; б - герметичного асинхронного двигателя 
Гринфельд Г. М., Голоколос Д. А.

ПАРАМЕТРИЧЕСКАЯ КОРРЕКЦИЯ СИСТЕМЫ УПРАВЛЕНИЯ ГЕРМЕТИЧНОГО ЭЛЕКТРОМЕХАНИЧЕСКОГО

ПРЕОБРАЗОВАТЕЛЯ

\section{Выводы}

Предложен подход к выполнению параметрической коррекции системы управления электромеханическим преобразователем, обеспечивающий высокие показатели качества регулирования приводов с герметичными асинхронными двигателями.

Рассматриваемая методика, построенная на основе приведения схемы замещения герметичного АД к стандартной, позволяет компенсировать влияние экранирующего элемента.

\section{ЛИТЕРАТУРА}

1. Голоколос, Д. А. Влияние параметров экрана на характеристики капсулированных асинхронных электродвигателей / Д. А. Голоколос // Учёные записки Комсомольского-на-Амуре государственного технического университета. Науки о природе и технике. - 2014. - № IV-1(20). - С. 1-4.

2. Чиликин, М. Г. Теория автоматизированного электропривода / М. Г. Чиликин, В. И. Ключев, А. С. Сандлер. - М.: Энергия, 1979.

3. Уайт, Д. С. Электромеханическое преобразование энергии / Д. С. Уайт, Г. Х. Вудсон. - Л.: Энергия, 1964.

4. Соколовский, Г. Г. Электроприводы переменного тока с частотным регулированием / Г. Г. Соколовский. - М.: Академия, 2006.

5. Копылов, И. П. Математическое моделирование электрических машин / И. П. Копылов. - М.: Высш. шк., 2001.

6. Рудаков, В. В. Системы управления электроприводов. Прямое управление моментом в электроприводе переменного тока: учеб. пособие / В. В. Рудаков, А. Е. Козярук. - СПб., 2007.

7. Народницкий, А. Г. Современное и перспективное алгоритмическое обеспечение частотно-регулируемых электроприводов / А. Г. Народницкий. - СПб.: Электротехническая компания, 2004. 